\title{
Record-High Secret Key Rate for Joint Classical and Quantum Transmission Over a 37-
} Core Fiber

da Lio, Beatrice; Bacco, Davide; Cozzolino, Daniele; Da Ros, Francesco; Guo, Xueshi; Ding, Yunhong; Sasaki, Yusuke; Aikawa, Kazuhiko; Miki, Shigehito; Terai, Hirotaka

Total number of authors:

17

Published in:

Proceedings of 2018 IEEE Photonics Conference

Link to article, DOI:

10.1109/IPCon.2018.8527341

Publication date:

2018

Document Version

Peer reviewed version

Link back to DTU Orbit

Citation $(A P A)$ :

da Lio, B., Bacco, D., Cozzolino, D., Da Ros, F., Guo, X., Ding, Y., Sasaki, Y., Aikawa, K., Miki, S., Terai, H., Yamashita, T., Neergaard-Nielsen, J. S., Galili, M., Rottwitt, K., Andersen, U. L., Oxenløwe, L. K., \& Morioka, T. (2018). Record-High Secret Key Rate for Joint Classical and Quantum Transmission Over a 37-Core Fiber. In Proceedings of 2018 IEEE Photonics Conference IEEE. https://doi.org/10.1109/IPCon.2018.8527341

\section{General rights}

Copyright and moral rights for the publications made accessible in the public portal are retained by the authors and/or other copyright owners and it is a condition of accessing publications that users recognise and abide by the legal requirements associated with these rights.

- Users may download and print one copy of any publication from the public portal for the purpose of private study or research.

- You may not further distribute the material or use it for any profit-making activity or commercial gain

- You may freely distribute the URL identifying the publication in the public portal 


\title{
Record-high secret key rate for joint classical and quantum transmission over a 37-core fiber
}

\author{
Beatrice Da Lio ${ }^{1}$, Davide Bacco ${ }^{1}$, Daniele Cozzolino ${ }^{1}$, Francesco Da Ros ${ }^{1}$, Xueshi Guo ${ }^{2}$, Yunhong Ding ${ }^{1}$, \\ Yusuke Sasaki ${ }^{3}$, Kazuhiko Aikawa ${ }^{3}$, Shigehito Miki ${ }^{4}$, Hirotaka Terai ${ }^{4}$, Taro Yamashita ${ }^{5}$, Jonas S. Neergaard- \\ Nielsen $^{2}$, Michael Galili ${ }^{1}$, Karsten Rottwitt ${ }^{1}$, Ulrik L. Andersen ${ }^{2}$, Leif K. Oxenløwe $^{1}$, Toshio Morioka ${ }^{1}$ \\ ${ }^{l}$ CoE SPOC, DTU Fotonik, Dep.of Photonics Eng., Technical University of Denmark, Ørsteds Plads Building 340, 2800 Kgs. Lyngby, Denmark \\ ${ }^{2}$ Center for Macroscopic Quantum States (bigQ), Dep. of Physics, Technical University of Denmark, Building 309, 2800 Kgs. Lyngby, Denmark \\ ${ }^{3}$ Advanced Technology Laboratory, Fujikura Ltd., 1440, Mutsuzaki, Sakura, Chiba, 285-8550, Japan \\ ${ }^{4}$ Advanced ICT Res. Inst., National Institute of Information and Communications Technology, 588-2 Iwaoka, Nishi-ku, Kobe, 651-2492, Japan \\ ${ }^{5}$ Department of Electronics, Graduate School of Engineering, Nagoya University, Furo-cho, Chikusa-ku, Nagoya, 464-8603, Japan \\ bdali@fotonik.dtu.dk,dabac@fotonik.dtu.dk
}

\begin{abstract}
We overcome the low secret key rates of current quantum communication systems using space division multiplexing in a heterogeneous 37-core fiber. We demonstrate record-high rates of $107 \mathrm{Mb} / \mathrm{s}$ for the quantum channel alone and of $65 \mathrm{Mb} / \mathrm{s}$ when copropagating with $370 \mathrm{~Gb} / \mathrm{s}$ classical data.
\end{abstract}

\section{INTRODUCTION}

Quantum key distribution (QKD) is being intensely studied as a means to securely share encryption keys for future absolute secure communication links [1]. The current critical limitations of QKD schemes are related to the short achievable propagation distances of single photon systems, the low obtainable key rates and the challenge of co-existence with classical data channels. In classical communication systems, we have recently seen great enhancements in total data capacity using space-division multiplexing (SDM) [2]. Uncoupled heterogeneous multicore fibers (MCFs) use single-mode cores with low cross-talk between the spatial channels, enabling multiple-input/multiple-output (MIMO)-free ultra-high capacity classical communication demonstrations [3-4]. The high isolation between the cores in these MCFs also renders them very useful for quantum applications, such as for enhancing the secret key rates in QKD systems either by creating highdimensional keys (qudits) or by multiplexing keys to higher rates [5-7]. In this paper, we propose to use independent cores of a recently developed MCF counting the highest number of cores achieved to date, 37 [8], to transmit parallel QKD keys to reach a recordhigh secret key rate. With all 37 cores fully lit by quantum signals, we achieve a joint secret key rate of $107 \mathrm{Mb} / \mathrm{s}$, more than 4 times higher than the previous record of $26 \mathrm{Mb} / \mathrm{s}$ [9]. Moreover, we demonstrate the robustness of our scheme by transmitting quantum channels together with classical channels: $10 \mathrm{~Gb} / \mathrm{s}$ through all 37 cores for a total co-propagating classical data rate of $370 \mathrm{~Gb} / \mathrm{s}$. With all 37 cores loaded with a classical and a quantum channel, the achieved secure key rate becomes $65 \mathrm{Mb} / \mathrm{s}$. This rate is higher than any other QKD-only reports to date, and 30 times higher than the current record for QKD-classical cotransmission [10-12]. The record-high rates achieved in this work are summarized in Fig. 1 a), showing the significant improvement compared to state of the art.
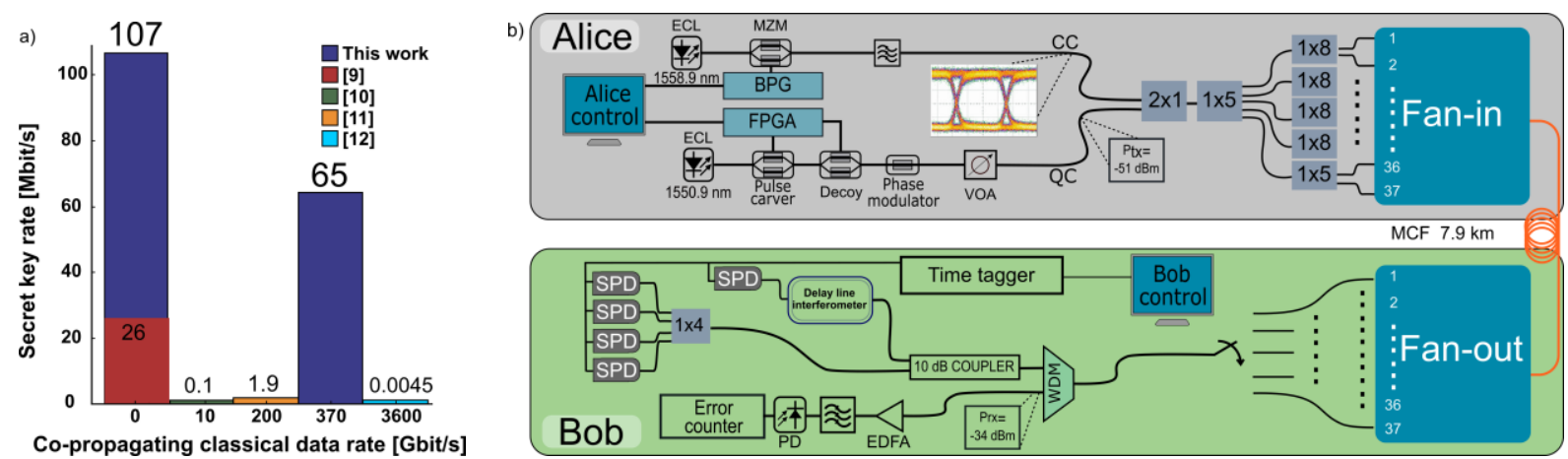

Fig. 1: a) Secret key rate as a function of co-propagating classical data rates achieved in the state of the art. b) Experimental setup. Top: Alice, the transmitter. Bottom: Bob, the receiver. FPGA: field programmable gate array; MZM: Mach-Zehnder modulator; OBPF: optical band-pass filter; CC: classical channel; QC: quantum channel. 

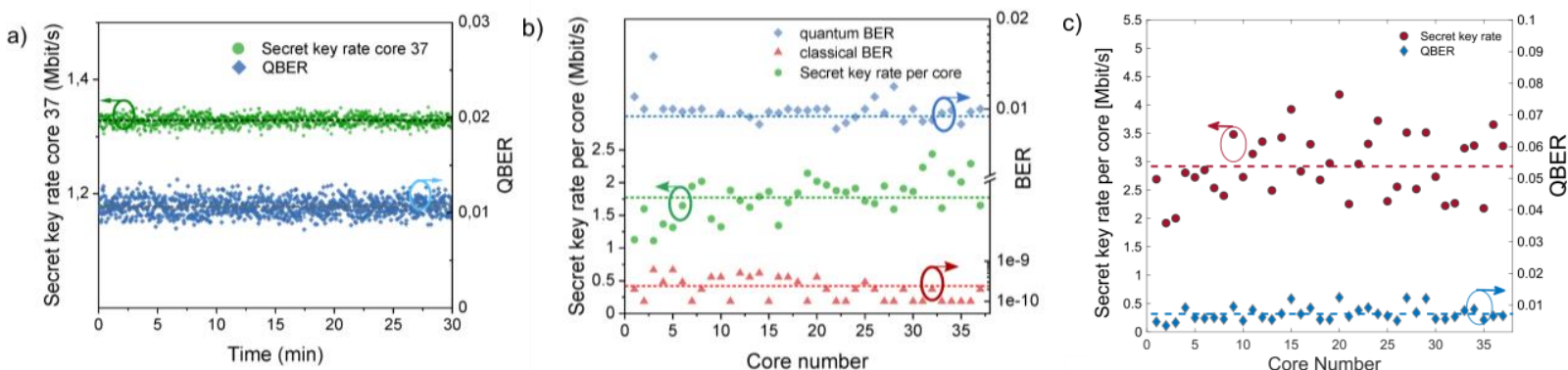

Fig. 2: a) Longterm stability of secret key rate and quantum bit error rate in core 37, in the QKD-classical co-propagation case. b) Generated secret key rate and measured quantum and classical bit error rates per core, in the QKD-classical co-propagation case. c) Generated secret key rate and measured quantum bit error rate per core, for QKD-only transmission scenario.

\section{EXPERIMENTAL SETUP AND RESULTS}

The experimental setup is shown in Fig. 1 b). A 7.9 km 37-core uncoupled heterogeneous MCF [8] is used to enhance the secret key rate through SDM. As QKD protocol, we implement the three states time bin BB84 1-decoy state protocol with finite key analysis [13-14]. A train of pulses is generated by carving a continuous wave laser at $1550.9 \mathrm{~nm}$ by an intensity modulator (IM) controlled by an FPGA board. A second IM is used to randomly prepare the quantum states $(595 \mathrm{MHz}$ repetition rate, adding data according to the chosen QKD protocol). A variable optical attenuator (VOA) and cascaded beam splitter are used to reduce the power to the quantum regime $(-74 \mathrm{dBm}$, i.e. an average of 0.1 photons per pulse) and to split into 37 different paths. $10 \mathrm{~Gb} / \mathrm{s}$ classical on-off keying signals at $1558.9 \mathrm{~nm}$ are simultaneously injected into all cores of the MCF. At the receiver, a wavelength division multiplexing (WDM) filter is used to separate classical from quantum channels. The classical channel is detected using a preamplifier and a photodetector, and the bit error rate (BER) is measured for all channels. A free-space delay line interferometer (visibility $\mathrm{V}=0.98$ ) is used for eavesdropping-checking in the quantum channel. We use superconductive nanowire single photon detectors (SN-SPDs) with around $60 \%$ efficiency, dark counts of $30 \mathrm{~Hz}$, connected to a time tagger unit acquiring the time of arrival of the photons. The measured bit error rate for the quantum channel (QBER) and achieved secret key rate for core 37 is shown in Fig. 2 a) when measured over 30 minutes and with co-transmission of a classical channel. An average secret key rate of 1.32

This work was supported by: SPOC Centre for Silicon Photonics for Optical Communications (ref DNRF123); bigQ Center for Macroscopic Quantum States (ref DNRF142); People Programme (Marie Curie Actions FP7/2007-2013- n 609405); VILLUM FOUNDATION Young Investigator Programme; EU-Japan coordinated R\&D project SAFARI commissioned by the Ministry of Internal Affairs and Communications of Japan and EC Horizon 2020.
$\mathrm{Mb} / \mathrm{s}$ and an average QBER of 1E-2 is achieved, which is well below the requirements for the used QKD protocol. Fig. 2 b) shows results for all 37 cores in terms of secret key rate and quantum and classical BER with simultaneous co-propagation of a classical $10-\mathrm{Gb} / \mathrm{s}$ channel (with BER < 1E-9). The average secret key rate per core is $1.77 \mathrm{Mb} / \mathrm{s}$ yielding a total rate of $65 \mathrm{Mb} / \mathrm{s}$. The measured BER for both classical and quantum channels are on average 2.4E-10 BER and 9.5E-3 QBER, respectively, being lower than standard requirements in both cases. When the classical channels are switched off, we achieve a successful transmission of a secret key rate of $2.9 \mathrm{Mb} / \mathrm{s}$ per core on average, resulting in a total rate of $107 \mathrm{Mb} / \mathrm{s}$, with average QBER of 7.2E-3, as shown in Fig. 2 c).

\section{CONCLUSIONS}

We demonstrated record-high secret key rates $(65 \mathrm{Mb} / \mathrm{s}$ for 37 classical and quantum channels co-transmitted, and $107 \mathrm{Mb} / \mathrm{s}$ for QKD-only) enabled by parallel transmission of quantum keys in a 37-core fiber.

\section{REFERENCES}

[1] J. Yin et al., Science 356, pp 1140-1144 (2017)

[2] D. Soma et al., ECOC 2017, Postdeadline paper Th.PDP.A.1

[3] T. Kobayashi et al, OFC 2017, Postdeadline paper Th5B.1.

[4] B. Puttnam et al, ECOC 2015, Postdeadline paper PDP.3.1.

[5] Y. Ding et al., npj Quantum Information 3, 25 (2017)

[6] D. Bacco et al., Scientific Reports 7: 12459 (2017)

[7] S. Pirandola et al., Nature Communications 8, 15043 (2017)

[8] Y. Sasaki et al., OFC 2017, Th1H.2 (2017)

[9] N. T. Islam et al., Science Advances 3, 11 e1701491 (2017)

[10] J. F. Dynes et al., Opt. Exp. 24, 8 pp8081-8087 (2016)

[11] J. F. Dynes et al., Scientific Reports 6: 35149 (2016)

[12] Y. Mao et al., Opt. Exp. 26, 5 pp6010-6020 (2018)

[13] D. Rusca et al., Applied Physics Letters 17,112 (2018)

[14] A. Boaron et al., arXiv:1807.03222 (2018) 